\title{
Social determinants of the impact of hospital management boards on quality management: a study of 109 European hospitals using a parsonian approach
}

Holger Pfaff $^{1 *}$ (D, Antje Hammer ${ }^{1}$, Marta Ballester ${ }^{2,3,4}$, Kristina Schubin ${ }^{1}$, Michael Swora ${ }^{1}$ and Rosa Sunol ${ }^{2,3,4}$

\begin{abstract}
Background: The consolidated framework for implementation research states that personal leadership matters in quality management implementation. However, it remains to be answered which characteristics of plural leadership in hospital management boards make them impactful. The present study focuses on social determinants of implementation power of hospital boards using Talcott Parsons' sociological concept of adaptation, goal attainment, integration, and latency (AGIL), focusing on the G (goal attainment) and I (integration) factors of this concept. The study aims to test the hypothesis that hospitals with management boards that are oriented toward the quality goal (G) and socially integrated (I) (GI boards) are better at implementing quality management than hospitals with boards lacking these characteristics (non-Gl boards).
\end{abstract}

Methods: A cross-sectional mixed-method design was used for data collection in 109 randomly selected hospitals in seven European countries. Data is based on the study "Deepening our understanding of quality improvement in Europe" (DUQUE). We used responses from (a) hospitals' chief executive officers to measure the variable social integration and the variable quality orientation of the board and (b) responses from quality managers to measure the degree of implementation of the quality management system. We developed the $\mathrm{Gl}$ index measuring the combination of goal-orientation and integration. A multiple linear regression analysis was performed.

Results: Hospitals with management boards that are quality oriented and socially integrated (GI boards) had significantly higher scores on the quality management system index than hospitals with boards scoring low on these features, when controlled for several context factors.

Conclusions: Our findings suggest that the implementation power of hospital management boards is higher if there is a sense of unity and purpose within the boards. Thus, to improve quality management, it could be worthwhile to increase boards' social capital and to increase time designated for quality management in board meetings.

Keywords: Quality management, Implementation power, Leadership, Hospital management board, Social capital, Top management team, Consolidated framework for implementation research

\footnotetext{
* Correspondence: holger.pfaff@uk-koeln.de

'Institute for Medical Sociology, Health Services Research and Rehabilitation

Science, Faculty of Human Science, Faculty of Medicine, University of

Cologne, Eupener Strasse 129, 50933 Cologne, Germany

Full list of author information is available at the end of the article
}

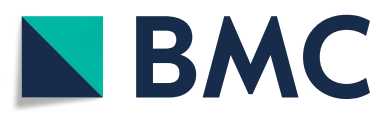

(c) The Author(s). 2021 Open Access This article is licensed under a Creative Commons Attribution 4.0 International License, which permits use, sharing, adaptation, distribution and reproduction in any medium or format, as long as you give appropriate credit to the original author(s) and the source, provide a link to the Creative Commons licence, and indicate if changes were made. The images or other third party material in this article are included in the article's Creative Commons licence, unless indicated otherwise in a credit line to the material. If material is not included in the article's Creative Commons licence and your intended use is not permitted by statutory regulation or exceeds the permitted use, you will need to obtain permission directly from the copyright holder. To view a copy of this licence, visit http://creativecommons.org/licenses/by/4.0/ The Creative Commons Public Domain Dedication waiver (http://creativecommons.org/publicdomain/zero/1.0/) applies to the data made available in this article, unless otherwise stated in a credit line to the data. 


\section{Background}

The consolidated framework for implementation research (CFIR) states that personal leadership is an important contextual determinant for the implementation of quality management [1, 2]. However, leadership may be plural [3], as is the case in hospital management boards (HMBs). It remains to be answered which features of plural leadership increase the power of a HMB to implement quality management throughout the hospital.

Research on success characteristics of HMBs provides insights into the importance of the structural characteristics of HMBs. These studies often examine the direct relationship between HMBs and health care quality [47], disregarding the mechanisms that transform the political will of plural leaders into positive health care outcomes. The implementation of quality management systems in the hospitals can potentially mediate between HMBs and patient care quality. The CFIR underscores the importance of leadership for implementation. We aim to improve this hypothesis by connecting it with social theory. Frameworks for implementation research, such as CFIR, focus on variables rather than theories; however, we are convinced that we have to use theories to explain how these variables function. Thus, we propose a framework-plus-theory approach in quality research. The CFIR states that leadership commitment is essential for the implementation of innovations and evidence-based practices [2]. We specify this hypothesis using the well-established sociological concept of AGIL by Talcott Parsons [8].

The AGIL scheme [8] assumes that social systems - such as HMBs - have to fulfill four central functions to survive and be productive: adaptation (A), goal attainment (G), integration (I), and latency (L). Because the function "goal attainment" is closely linked to the CFIR notion of leadership commitment, we focus on the factor goal attainment ( $G$ factor). However, our assumption is that the HMBs' plural leadership commitment alone is not sufficient to exert power and influence from the top level (HMB) to the middle and lower levels of hospital management to fully implement a hospital-wide quality management system. Additionally, HMBs need a certain sense of unity and agreement within the top management team. Therefore, based on the AGIL concept, we add the factor social integration (I factor) to the G factor. Social integration makes a group of leaders act like a single leader, making plural leadership a united leadership. The integration factor is advantageous because it allows HMBs to speak with one voice, reach balanced and mature decisions, and communicate the committed decisions via committed board members to the stakeholders they represent (e.g., physicians, nurses, and administrators).
Based on the GI model of system performance $[9,10]$, we define the GI factor as follows: The GI factor is given in a group if two conditions are met: (1) strong actions of the group to attain specific goals (G factor) and (2) a high degree of social integration within the group (I factor). Top management teams with the GI factor have a sense of unity and purpose, which makes them impactful. Therefore, our hypothesis is that HMBs, which combine high goal attainment with regard to quality (G) and high social integration within the board (I) (GI boards) have greater implementation power than HMBs with low integration and low quality goal orientation (non-GI boards).

\section{Methods}

We used data from the multicenter cross-sectional study "Deepening our understanding of quality improvement in Europe" (DUQuE) [11]. DUQuE fulfilled the ethical requirements for research projects as described in the seventh framework of EU Directorate-General Research. Ethics approval was granted through the Bioethical Committee of the Department of Health of the Government of Catalonia, Spain. Data collection in each country complied with confidentiality requirements according to the national legislation or standards of practice of that country.

Patient records and other information was anonymized and de-identified prior to analysis. The inclusion criteria for hospitals were as follows: (a) having 120 beds or more, (b) delivering care for four medical conditions acute myocardial infection, stroke, hip fracture, and delivery and (c) having a HMB with at least 2 but no more than 25 board members. The exclusion criterion was missing data in the variables used in the regression analysis. An analysis of the missing data showed only minor differences between the sample obtained after applying only the inclusion criteria $(\mathrm{N}=152)$ and the sample obtained after applying the exclusion criterion as well $(\mathrm{N}=$ 109). There were no or only minor differences in the distribution of the following variables: public hospitals (full sample: $82.9 \%$ vs. missing-data-free sample: $82.6 \%$ ), social capital (mean: 3.27 vs. 3.25), quality orientation (mean: 3.86 vs. 3.94 ), and GI index (GI hospitals: $29.5 \%$ vs. $30.3 \%$ ). There were differences regarding hospital size (hospitals with more than 500 beds: $45.4 \%$ vs. $51.3 \%$ ) and small differences regarding teaching status $(40.1 \%$ vs. $42.2 \%)$. Data collection was performed in 109 randomly selected hospitals in the Czech Republic, France, Germany, Poland, Portugal, Spain, and Turkey. In the present study, we used the responses of the hospitals' chief executive officers (CEOs) and quality managers obtained through web-based questionnaires. Data were collected between May 2011 and February 2012. 
Items used in the questionnaire were either newly developed in English (e.g. quality management system index) or translated from existing measures into English (e.g. social capital scale) or adapted. The final questionnaire has been translated into seven languages following a standard protocol for a forward-backward translation process supervised by local country coordinators [12, 13]. We used a 46-item quality management system index as the dependent variable measuring the degree of implementation of quality management systems in hospitals from the perspective of the quality managers. This index was the sum of scores on the following nine subscales: quality policy documents (three items), quality monitoring by the board (five items), training of professionals (nine items), formal protocols for infection control (five items), formal protocols for medication and patient handling (four items), analyzing performance of care processes (eight items), analyzing performance of professionals (three items), analyzing feedback and patient experiences (three items), and evaluating results (six items). Eight of the nine subscales of this index had sufficient reliability (Cronbach's alpha: 0.70-0.88), whereas one subscale - analyzing feedback and patient experiences - did not. To ensure content validity, we decided to include this subscale in the quality management system index as well. For details, see Wagner et al. [12]. We used the averaged composite score for the nine subscales ranging from 0 to 27 points.

To measure the first component of the GI index - the G factor - we built the index "hospital board quality goal orientation" as a surrogate variable. Based on the empirical work of Botje and colleagues concerning quality orientation of hospital management boards [14] we measured the goal attainment index by asking the CEOs (a) to assess how frequently quality performance was an item on the executive board's agenda during the last year (never [1], in a few meetings [2], in most meetings [3], or in every meeting [4]) and (b) to assess what percentage of the hospital (management) board's meeting time was typically spent on issues of quality performance (10\% or less [1], $11-20 \%$ [2], $21-30 \%$ [3], 31-40\% [4], and greater than $40 \%$ [5]). This index was built by adding the answers of these two questions. On the basis of this index, we then constructed a dichotomous variable using the tercile split method, creating a binary variable: low quality orientation (low and medium tercile [0]) and high quality orientation (upper tercile [1]). We used the upper tercile split method and not the median split method based on the assumption that it is necessary to have a strong goal orientation in the HMBs to have an impact on the followers across different management levels.

To measure the second component of the GI index the I factor - we used the scale "social capital within the hospital management board" [15] as a proxy for social integration of the board. It was derived from a validated scale called social capital of healthcare organizations from employees' perspective (SOCAPO-E), which has been used in previous studies [16, 17]. For this study, the six items of this scale were adapted in order to measure social capital among hospital management board members (SOCAPO-B). The scale consists of six items (Cronbach's alpha: 0.91) measuring the amount of unity and agreement, common values, mutual trust, mutual helpfulness, "we" feeling, and good social climate in the HMBs. The scale reflects central features of the communal aspects of social capital [17-19]. We used a 4-point Likert scale from "strongly disagree" (1) to "strongly agree" (4). To construct the dichotomous variable "integration index", which separates HMBs scoring high in social capital (1) from those scoring low (0). Again, we used the upper tercile split and not the median split method based on the assumption that for having adequate implementation power, a quite high amount of unity and cohesion within HMBs is needed.

Based on the goal-integration model $[9 ; 10]$ we developed a GI index by adding both dichotomous variables, resulting in an index with three values: 0 (HBM with low quality goal orientation and low social capital), 1 (HBM low in one of these two variables), and 2 (HBM high in both variables). Thus, we did not differentiate between the other two combinations - HMB with high quality goal orientation but without high social capital and HMB without high quality goal orientation but with high social capital - because both combinations are considered equivalent in promoting collective action. We used board size, ownership, hospital size, teaching status of hospital, and country as control variables. For the multivariate analysis, we used multiple linear regression with a restricted and a full model using IBM SPSS STATISTICS version 26.

\section{Results}

\section{Sample characteristics and descriptive statistics}

The dataset included in this analysis contained only hospitals with complete data on all variables used in the multiple regression model $(\mathrm{N}=109)$. The sample included seven countries (see Table 1); 82.6\% were public hospitals, and $42.2 \%$ were teaching hospitals. Approximately $11 \%$ of the hospitals had less than 200 beds; $37.6 \%$ had $200-500$ beds; $33.9 \%$ had $501-1000$ beds; and $17.4 \%$ had more than 1000 beds. The average number of HMB members in our sample was $7.6(\mathrm{SD}=4.1)$. The mean of the social capital scale - as one component of the GI index - was $3.3(\mathrm{SD}=0.61)$, measured on a scale ranging from 1 to 4 . The dichotomization of this scale using an upper tercile split showed that $64.2 \%$ and $35.8 \%$ of the hospitals, respectively, had low and high $\mathrm{HMB}$ 
Table 1 Percentage of the hospitals in European countries participating in DUQUE used in the analysis $(N=109)$

\begin{tabular}{llrr}
\hline & N & \% \\
\hline Country & Czech Republic & 19 & 17.4 \\
& France & 17 & 15.6 \\
Germany & 7 & 6.4 \\
Poland & 17 & 15.6 \\
Portugal & 19 & 17.4 \\
Spain & 19 & 17.4 \\
Turkey & 11 & 10.1 \\
& Total & 109 & 100.0 \\
\hline
\end{tabular}

social capital. The mean of the hospital board quality goal orientation index - the other component of the GI index - was $3.9(\mathrm{SD}=1.61)$, measured on a scale ranging from 0 to 7. The dichotomization of this scale using an upper tercile split indicated that $58.7 \%$ and $41.3 \%$ of the hospitals, respectively, showed low and high HMB quality goal orientation. The creation of the goal-integration index led to three GI levels, with $40.4 \%$ of the HMBs $(\mathrm{N}=44)$ on the lowest level, $42.2 \%(\mathrm{~N}=46)$ on the middle level, and $17.4 \%(\mathrm{~N}=19)$ on the highest level (see Table 2). Moreover, the average mean of the outcome variable quality management system index was 19.3 $(\mathrm{SD}=4.6)$ on a scale ranging from 0 to 27 .

\section{Regression analyses}

Checking for outliers with Cook's distance proved to be negative, indicating no outliers. The results of the linear regression analysis (see Table 3 ) indicate a significant association between the boards' GI index and the quality management system index. Compared with the restricted model containing the control variables

Table 2 Goal-integration (Gl) index ( $N=109)$

\begin{tabular}{lrc}
\hline Characteristic of Hospital Management Board (HMB) & N & $\%$ \\
\hline Quality goal orientation of HMB (G index) & & \\
0 - HMB low in quality goal orientation & 64 & 58.7 \\
1 - HMB high in quality goal orientation & 45 & 41.3 \\
Total & 109 & 100 \\
Social capital of HMB (I index) & & \\
0 - HMB low in social capital & 70 & 64.2 \\
1 - HMB high in social capital & 39 & 35.8 \\
Total & 109 & 100 \\
Gl index & & \\
0 - HMB low in social capital and quality goal orientation & 44 & 40.4 \\
1 - HMB low in social capital OR quality goal orientation & 46 & 42.2 \\
2 - HMB high in social capital and quality goal orientation & 19 & 17.4 \\
Total & 109 & 100 \\
\hline
\end{tabular}

(explained variance: 27,6\%; $p<0.001$ ), the introduction of the GI factor into the equation led to a significant increase of $5,7 \%$ in explained variance $(p<0.05)$; the full model explained $33 \%$ of the variance in the quality management system index. Hospitals with boards high in the GI index had significantly better scores on the quality management system index than hospitals with boards low in quality goal orientation and in social capital (reference group: non-GI-board hospitals; $p<0.01$ ). The results also showed that hospitals that meet only one of both impact requirements - HMB quality goal orientation or HMB social capital - have an advantage over hospitals that fulfill none of the two requirements, but this advantage was not significant. We conducted regression diagnostics by checking the assumptions of linearity, variance homogeneity and normally distributed errors by means of scatter- and QQ-plots of the standardized residuals, which proved to be well-behaved. Furthermore, there were no signs of multicollinearity as measured by the condition index.

\section{Discussion}

Implementation of quality management systems in hospitals is one of the central tasks in quality and safety. According to the CFIR, one way to promote this is using leadership commitment [2]. In the case of HMBs, leadership is plural leadership. The present study aimed to investigate which conditions foster the implementation power of plural leadership. To answer this question, we considered the HMB as a social system and used Parsons' social system theory.

On the basis of Parsons' sociological concept of AGIL, we proposed that the combination of goal attainment (G) and social integration (I) - the GI factor - is a social precondition for any collective endeavor such as the implementation of a hospital-wide quality management system. In general, our empirical results support the hypothesis that, in European hospitals, quality management strategies are fully implemented more often if the hospitals are managed by hospital boards with a high GI factor. After adjusting for various potential confounding variables, hospitals with GI boards showed a higher score on the quality management system index than hospitals led by non-GI boards. Hypothetically, this means that hospitals could realize an absolute improvement of 3.3 points on the 27 -point quality management system index (11\% improvement) if there is strong emphasis on quality performance and a sense of unity within the HMBs. There was no significant increase compared with the reference group (low GI-board hospital) if the HMB either had quality orientation only (G) or a sense of unity only (I). Therefore, having a board with only one of these success factors does not seem 
Table 3 Regression analysis with quality management system index as dependent variable $(N=109)$

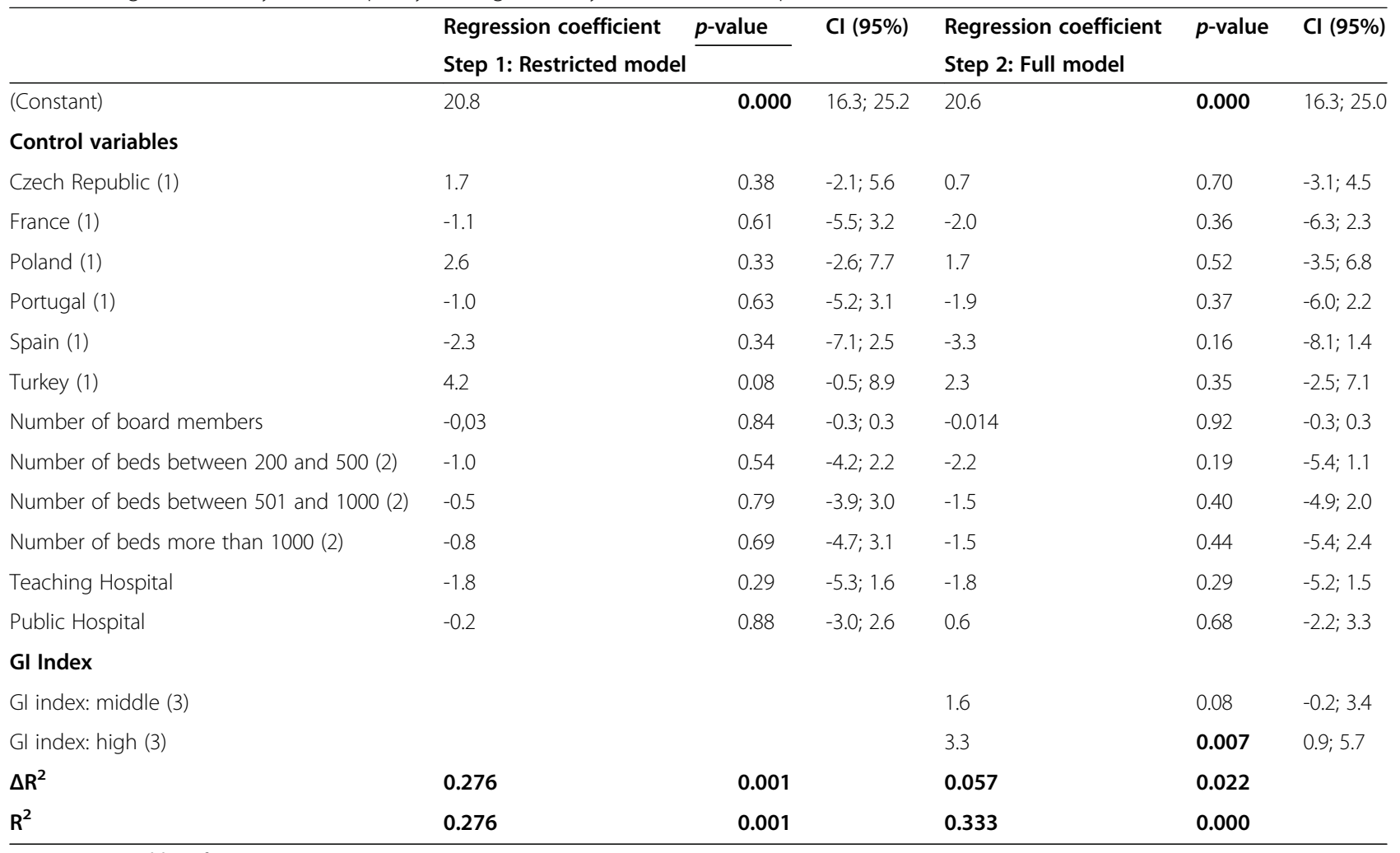

(1) Dummy variable: reference category: Germany

(2) Dummy variable: reference category: number of beds lower than 200

(3) Dummy variable: reference category: GI index = low

sufficient. The possible explanations for this finding need to be explored.

Our results correspond with the upper echelons theory [20]. This theory states that certain characteristics of top management teams influence the decision making of the top team itself as well as the decisions and behaviors of others outside the team, especially employees in the organization [20].

Thus, we have two types of HMB impact: (a) an inward impact and (b) an outward impact. One inward impact is that HMBs with a high degree of social integration can make quick collective decisions owing to shared values and a common will to reach agreement [21]. The other inward impact is that socially integrated HMBs are able to integrate different cultures and stakeholders within the HMB through group-bonding processes, leading to more culturally balanced decisions and less social conflicts within HMBs. Shared leadership studies support this perspective [22, 23].

An important outward impact of an integrated and committed HMB is its power to influence the lower hierarchical levels and its power to implement innovations on these levels. This implementation power depends on the formal power of the HMB to make decisions and to lead the hospital. Moreover, this implementation power stems from the social contagion effect of committed groups. Social contagion describes the diffusion of emotions, cognitions, attitudes, or behaviors in groups, networks, and organizations [24]. Studies showed that leaders' mood and passion [25] as well as their commitment towards a certain goal (e.g., quality goal) are contagious to the followers [26, 27]. Furthermore, the implementation power is based on the unity of the group. This unity bundles together the individual energies of the board members forming a cohesive top management team with high social energy that can be used to influence people (I: integration). Additionally, the implementation power stems from the role model function of a quality-oriented HMB. Qualitystriving boards are an inspiring example for attaining quality goals throughout the hospital on all hierarchical levels (G: goal attainment). The Ohio model of leadership further supports this informal power perspective [28]. The Ohio model states that successful leadership combines two dimensions: (a) consideration (fostering the quality of the social relationships between leaders and followers) and (b) initiating structure (the degree to which a leader is oriented toward goal attainment) [29]. The first dimension relates to the I factor in the AGIL concept, and the second dimension relates to the $\mathrm{G}$ factor. 
Implementation power is important for transferring quality practices to all hospital levels because the implementation of quality-oriented structures and processes affects employees with vested interests and involves activating resources controlled by other managers [30]. Empirical results suggest that "in order to have effective strategy implementation processes (...) top management teams first need to minimize within-team fragmentation pressures to achieve integration" [31]. Therefore, strengthening the social capital of HMBs by improving the team climate is important to foster the implementation power of the board.

\section{Strengths and limitations}

The presented data are based on a cross-national, mixed-method study in seven European countries. We mostly used validated scales. Moreover, we used different sources of data for the GI index (CEO questionnaire) and for the quality management system index (quality manager questionnaire) to diminish the risk of common method variance bias [32]. However, the present analyses have certain limitations that are worth mentioning. First, the study is based on a cross-sectional design and does not allow us to draw causal conclusions. Future studies should strive for longitudinal designs. Second, social capital, quality goal orientation, and quality management system are self-reported measures based on the questionnaires answered by the CEOs and quality managers. These variables are not based on objective measurements (e.g. observations), which would be an additional goal for future studies. Therefore, they represent the perception of these professionals, which should be considered when interpreting the results. Third, the two-item index "hospital management board quality goal orientation" is not a validated measure yet. However, this index is based on quality management research and has been developed and used in a previous study as a reliable, fact-based measure [14]. By dichotomizing this index, we additionally facilitated the robustness of the measure. Fourth, one could criticize the key informant approach used; we chose this approach owing to its practicability. Nevertheless, the use of key informants is common practice in business research and health services research. Fifth, despite high response rates, our results are restricted to 109 hospitals because of missing values in the quality management system index. Further analysis showed no significant differences between the original sample and the analyzed sample of 109 hospitals. Sixth, we considered most potential confounders, but uncontrolled confounding cannot be completely eliminated. Seventh, despite our efforts to use a validated approach for backward-forward translation [13], we cannot fully rule out the possibility that the meaning of the items used might differ across national cultures and regulatory environments.

\section{Conclusions}

To the best of our knowledge, this is the first large-scale study that explored two possible social determinants of the impact of hospital boards on quality management: quality goal orientation ( $\mathrm{G}$ factor) and social integration (I factor). Due to the limitations of this study, we have to be careful with conclusions. However, we can state that this study could be regarded as a hypothesis generating study. Hence, the present study improves the consolidated framework for implementation research by generating the hypothesis that shared commitment towards quality performance combined with social integration within HMBs could possibly increase the power of the HMBs to implement quality management systems within the hospital. Regarding future research, longitudinal studies are required to further examine the relationship between HMB characteristics and implementation of quality management systems. Regarding quality management practices, results indicate tentatively that two practical measures could strengthen the implementation of evidence-based quality in hospitals: (1) fostering the social capital in HMBs using team development and (2) reserving time to discuss quality on the agenda of the board meetings.

\section{Abbreviations \\ CFIR: Consolidated Framework for Implementation Research; GI: Goal- Integration; DUQUE: Deepening our Understanding of Quality improvement in Europe; CEOs: Chief Executive Officers; HMBs: Hospital Management Boards; AGIL: Adaptation, Goal attainment, Integration, Latency; SD: Standard Deviation; Cl: Confidence Interval}

\section{Acknowledgements}

We thank the experts, country coordinators and participating hospitals of the DUQuE project for their valuable advice on the measurement instruments, and the respondents for their effort and time to fill in the questionnaires.

\section{Authors' contributions}

HP contributed to funding acquisition, conceptualization, project administration, methodology, investigation, software, data curation, formal analysis, original draft preparation, reviewing, and editing. AH contributed to project administration, investigation, data curation, and formal analysis. MB contributed to conceptualization, reviewing, and editing. KS contributed to formal analysis, reviewing, and editing. MS contributed to methodology and formal analysis. RS contributed to funding acquisition, project administration, supervision, conceptualization, validation, reviewing, and editing. The author(s) read and approved the final manuscript.

\section{Funding}

This work was supported by the European Commission's Seventh Framework Program FP7/2007-2013 [ec.europa.eu/index_en.htm] under the grant agreement number 241822. The funders had no role in study design, data collection and analysis, decision to publish, or preparation of the manuscript. Open Access funding enabled and organized by Projekt DEAL.

Availability of data and materials

The datasets used and/or analyzed during the present study are available upon reasonable request. Please send your request to Rosa Sunol (rsunol@fadq.org; ORCID: 0000-0003-1902-833X). 


\section{Ethics approval and consent to participate}

This was a secondary data analysis of the DUQUE data. The ethics approval for the DUQUE data was mentioned in the method section. As a member of the project team no administrative permissions were required to access and use the data described in this study.

\section{Consent for publication}

Not applicable.

\section{Competing interests}

The corresponding author, Holger Pfaff, is a member of the editorial board of this journal. The co-authors declare that they have no competing interests.

\section{Author details}

'Institute for Medical Sociology, Health Services Research and Rehabilitation Science, Faculty of Human Science, Faculty of Medicine, University of Cologne, Eupener Strasse 129, 50933 Cologne, Germany. ${ }^{2}$ Avedis Donabedian Research Institute (FAD), Universitat Autònoma de Barcelona, Barcelona, Spain. ${ }^{3}$ Universitat Autònoma de Barcelona, Barcelona, Spain. ${ }^{4}$ Red de Investigación en Servicios de Salud en Enfermedades Crónicas (REDISSEC), Barcelona, Spain

Received: 10 July 2020 Accepted: 29 December 2020

Published online: 19 January 2021

\section{References}

1. Taylor N, Clay-Williams R, Hogden E, et al. High performing hospitals: a qualitative systematic review of associated factors and practical strategies for improvement. BMC Health Services Research. 2015;15:244.

2. Damschroder $L$, Lowery JC. Evaluation of a large-scale weight management program using the consolidated framework for implementation research (CFIR). Implementation Science. 2013;8:51.

3. Denis J-L, Langley A, Sergi V. Leadership in the Plural. The Academy of Management Annals. 2012;6:211-83.

4. Jha A, Epstein A. Hospital governance and the quality of care. Health Aff. 2010:29:182-7

5. Botje D, Klazinga NS, Sunol R, et al. Is having quality as an item on the executive board agenda associated with the implementation of quality management systems in European hospitals: a quantitative analysis. Int J Qual Health Care. 2014:26:92-9.

6. Jiang HJ, Lockee $\mathrm{C}$, Bass $\mathrm{K}$, et al. Board engagement in quality: findings of a survey of hospital and system leaders. J Healthc Manag. 2008;53:121-34.

7. Berwick DM. Era 3 for Medicine and Health Care. JAMA. 2016:315:1329-30.

8. Parsons T, Smelser NJ. Economy and Society. London: Routledge; 1956.

9. Pfaff H. General performance requirements of health care organizations: the goal-integration model and the Gl factor: Discussion paper. https://kups.ub. uni-koeln.de/10124/ (18 December 2019, date last accessed).

10. Pfaff H, Braithwaite JA. A Parsonian Approach to Patient Safety: Transformational Leadership and Social Capital as Preconditions for Clinical Risk Management—the Gl Factor. Int J Environ Res Public Health. 2020;17: 3989.

11. Secanell M, Groene O, Arah OA, et al. Deepening our understanding of quality improvement in Europe (DUQuE): overview of a study of hospital quality management in seven countries. Int J Qual Health Care. 2014;26:515.

12. Wagner C, Groene O, Thompson CA, et al. Development and validation of an index to assess hospital quality management systems. Int J Qual Health Care. 2014:26:16-26.

13. Guillemin F, Bombardier C, Beaton D. Cross-cultural adaptation of healthrelated quality of life measures: literature review and proposed guidelines. J Clin Epidemiol. 1993;46(12):1417-32.

14. Botje D, Klazinga NS, Wagner C. To what degree is the governance of Dutch hospitals orientated towards quality in care? Does this really affect performance? Health Policy. 2013;113:134-41.

15. Hammer A, Arah OA, DerSarkissian M, et al. The relationship between social capital and quality management systems in European hospitals: A quantitative study. PLoS One. 2013;8(12):e85662. https://doi.org/10.1371/ journal.pone.0085662.

16. Ernstmann N, Ommen O, Driller E, et al. Social capital and risk management in nursing. J Nurs Care Qual. 2009;24:340-7.
17. Ansmann L, Hower Kl, Wirtz MA, et al. Measuring social capital of healthcare organizations reported by employees for creating positive workplaces validation of the SOCAPO-E instrument. BMC Health Services Research. 2020:20(1):1-10.

18. Putnam RD. Bowling alone: America's declining social capital. Journal of Democracy. 1995;6:65-78.

19. Cohen D, Prusak L. good company: How social capital makes organizations work. Boston: Harvard Business School Pr; 2001.

20. Hambrick DC. Upper echelons theory: An update. Acad Manag Rev. 2007;32: 334-43.

21. Combe IA, Carrington DJ. Leaders' sensemaking under crises: Emerging cognitive consensus over time within management teams. The Leadership Quarterly. 2015;26:307-22.

22. Rosengren K, Bondas T. Supporting "two-getherness": Assumption for nurse managers working in a shared leadership model. Intensive Critical Care Nursing. 2010;26:288-95.

23. Döös $M$, Vinell H, Knorring Mv. Going beyond "two-getherness": Nurse managers' experiences of working together in a leadership model where more than two share the same chair. Intensive Critical Care Nursing. 2017; 43:39-46.

24. Barsade SG, Coutifaris CGV, Pillemer J. Emotional contagion in organizational life. Research in Organizational Behavior. 2018;38:137-51.

25. Sy T, Choi JN. Contagious leaders and followers: Exploring multi-stage mood contagion in a leader activation and member propagation (LAMP) model. Theories of Cognitive Self-Regulation. 2013;122:127-40.

26. Shapiro A. Creating contagious commitment: Applying the tipping point to organizational change. 2nd ed. Hillsborough: Strategy Perspective; 2016.

27. Cabot H. 'Contagious' solidarity: reconfiguring care and citizenship in Greece's social clinics. Social Anthropology. 2016;24:152-66.

28. Stogdill RM, Coons AE, editors. Leader behavior: Its description and measurement. Columbus: Bureau of Business Research; 1957.

29. Judge TA, Piccolo RF, llies R. The forgotten ones? The validity of consideration and initiating structure in leadership research. J Appl Psychol. 2004;89:36.

30. Pfeffer J, Salancik GR. The external control of organizations: A resource dependence perspective. Stanford: Stanford Business Books; 2003.

31. Mistry S, Barrick M, Kirkman B, et al. TMT Strategy Implementation Tasks and Firm Performance: Teamwork Processes and Interdependence. In: Academy of Management Proceedings 2018;11418. DOl: https://doi.org/10.5465/ AMBPP.2018.11418abstract.

32. Podsakoff PM, MacKenzie SB, Lee J-Y, et al. Common method biases in behavioral research: A critical review of the literature and recommended remedies. J Appl Psychol. 2003;88:879-903.

\section{Publisher's Note}

Springer Nature remains neutral with regard to jurisdictional claims in published maps and institutional affiliations.

Ready to submit your research? Choose BMC and benefit from:

- fast, convenient online submission

- thorough peer review by experienced researchers in your field

- rapid publication on acceptance

- support for research data, including large and complex data types

- gold Open Access which fosters wider collaboration and increased citations

- maximum visibility for your research: over $100 \mathrm{M}$ website views per year

At $\mathrm{BMC}$, research is always in progress.

Learn more biomedcentral.com/submissions 